\title{
To establish a bicycle riding computer simulation model with preferable riding condition*
}

\author{
胡 祖武******, 李 傳房***
}

\begin{abstract}
Ergonomic considerations are important for a well-designed bicycle. The aim of our research is to evaluate the influence of seat height on the rider's physical factors, including anthropometry of the human body and joint angles, as well as psychological responses and subjective sensations. A questionnaire utilizing both Borg's scale and the Likert scale were used to determine riders' subjective opinions regarding preferable riding condition. The relationship between preferable riding condition and seat height was then established from the regression curve of the riders' subjective sensations with respect to the seat height. Using the regression curve, one can determine the preferable seat height for a comfortable riding posture. The results of this study can be used to develop a riding simulation model on a computer, which may benefit bicycle designers and manufacturers who are looking to improve the ergonomic design of their products. Research using CAD systems requires standard parameters of bicycle construction to set up a computer-simulated riding model. After creating a data bank of riders' physical factors and psychological responses, one can analyze the parameters of the preferable riding model with computer simulation, including seat height and height of handlebars.

(Key words : bicycle, preferable riding condition, computer simulation model)
\end{abstract}

\section{Introduction}

Although the development and manufacturing of bicycles has been going on for more than forty years in Taiwan, preferable riding condition and related human factors are still not well investigated. One of the papers with direct relevance to bicycle riding comfort mainly discussed the influence of gradient vibration surfaces on riding and maintenance of riding balance as some of the causes leading to physical discomfort ${ }^{1)}$. The other paper mentioned the specialty of bicycle construction with regard to the human body ${ }^{2)}$. Another article reported on calculations of relative human factors in bicycle riding and discussed referable size and angles of joints ${ }^{3)}$.

Some papers from the international literature do

* 受付 $: 2007$ 年 5 月 17 日 受理 $: 2007$ 年 10 月 12 日

** 朝陽科技大学設計研究所

Gradate Institate of Design, Chaoyang University of Technology

*** 雲林科技大学設計学研究所

Graduate School of Design, National Yunlin University of Science and Technology mention preferable bicycle riding condition. Two examples are mentioned below. The first paper examined the relation between the rider's related optimal size measures and joint angles ${ }^{4}$. The second paper developed a commercial bicycle selection and adjustment model and provided an evaluation on the usefulness of the model. The model allowed users to seat on an adjustable bicycle frame and changing the parameter relating to preferable riding condition including seat height, seat angle, seat tube slant angle, crank length and handlebar height. This model was utilized in a bicycle dealer's showroom to help consumers to find out their preferable range. The measurements data is useful in decision making on bicycle selection ${ }^{5}$. On the whole, these studies still lack stringent definitions on preferable riding condition.

As for the design of the frame of a bicycle, some schemes have used CAD systems, but none of them have united analysis of frame design with human factors. Our research will create criteria for the evaluation of bicycle design for comfortable riding, which will serve as a reference for and judgment of whether or not the frame design of a bicycle follows 
preferable values obtained from a computer simulation model. The following discussion of parameter design concerns frame design relative to a comfortable riding situation, and will help to determine the range of seat heights that correspond to preferable riding values.

Preferable riding condition is closely related to human factors. Long-duration-riding induces fatigue, and these subjective sensations must be considered in the design of a bicycle. For an ergonomic study, Borg's scale ${ }^{6)}$ and the Likert scale ${ }^{7)}$ have also been widely used for human psychological reactions, but rarely applied to human reactions while bicycle riding. This study utilizes the aforementioned techniques to establish a database of human physical factors and psychological responses that occur during bicycle riding. The database will be used to establish a computer simulation model for riding, in order to assist bicycle manufacturers in ergonomic design.

\section{Methods}

As for the human factors in bicycle riding, these can be approached in two directions, namely (1) physical factors, including anthropometry of the human body and joints angles, and (2) psychological responses, such as subjective sensations.

\section{2-1. Anthropometry of Bicycle Riding}

We have taken the opportunity to review the relative research about bicycles, looking for the preferable joint angles of the body, hands and feet. We also collected some human body dimensions database of different areas, such as Taiwan, Japan, American, France and German ${ }^{8 \sim 11}$. We will discuss how the size of the bicycle can be matched to the body, thereby determining a well-matched relationship between the rider and the bicycle.

\section{2-2. Subjective Sensation}

In this study, psychological responses are measured by a subjective sensation inventory. The preferable sensations in four parts of the riders' bodies (arms and hands, legs, hips, and trunk) were also obtained by use of the Likert scale. The Likert scale uses a descrip- tive five-point scale to label and distinguish different levels of rider comfort condition : comfortable, slight discomfort, medium discomfort, uncomfortable, and very uncomfortable. The subjective sensation was obtained by use of Borg's category-ratio scale which applies a percentage scale to divide the difference in comfort into various levels from comfortable to extremely uncomfortable. Borg's category-ratio scale was used for testing the subjective sensation in the present study since this scale is a ratio scale. To permit the total comfort sensation level to be precise, the value of this scale was increased 10 times (e.g. 0 is "Nothing at all", 30 is "uncomfortable" and 100 is "Very very uncomfortable"). The subject may decide which description in the scale best fits the level of comfort of various parts of his body, as well as the perceived overall comfort level.

To test psychological responses to riding a bicycle, we selected a generic bicycle type for the experiment. Seat and handlebar heights of the experimental bicycle are shown in Table 1 . The experimental riding time is 60 seconds. Eight subjects participated in the experiment. The range of these subjects' age is 19-23. All of these subjects are male. Before the experiment, the anthropometry of bicycle riding is conducted. The measured items include height, weight, thigh length, upper arm length, knee joint height of sitting, and elbow-grip length. The mean, standard deviation, minimum value and maximum value of each item are shown in Table 2. The pedaling speed was kept at around $15 \mathrm{~km} / \mathrm{h}$. After this riding experiment, we asked each rider about his subjective sensations.

\section{Results}

\section{3-1. Anthropometry of Bicycle Riding}

First we will discuss the relation of riding joint angles to the human factors of riding comfort. These

Tab. 1 Experimental conditions.

\begin{tabular}{lcccccc}
\hline & \multicolumn{3}{c}{ Seat height } & \multicolumn{3}{c}{ Handlebar height } \\
& S 1 & S 2 & S 3 & S 4 & H 1 & H 2 \\
\hline General bicycle & 562 & 618 & 674 & 730 & 968 & 1005 \\
\hline Unit : $\mathrm{mm}$ & & & & & &
\end{tabular}


Tab. 2 Subject's anthropometry of bicycle riding.

\begin{tabular}{lccc}
\hline Items & Height & Thigh length & $\begin{array}{c}\text { Knee joint } \\
\text { height of sitting }\end{array}$ \\
\hline Mean & 1734 & 454.9 & 441.6 \\
SD & 37 & 14.4 & 14.2 \\
Min & 1685 & 440 & 430 \\
Max & 1772 & 485 & 460 \\
\hline Items & Weight & Upper arm & Elbow-grip \\
& & length & length \\
\hline Mean & 70.89 & 355.1 & 365.9 \\
SD & 11.63 & 12.6 & 18.1 \\
Min & 57 & 333 & 340 \\
Max & 93.8 & 368 & 393 \\
\hline
\end{tabular}

unit: weight is $\mathrm{kg}$, and the other items are $\mathrm{mm}$

Tab. 3 Comparison of comfortable angle of body joints when riding bicycles.

\begin{tabular}{lcc}
\hline & $\begin{array}{c}\text { 1993 Juang } \\
\text { 5\%tile 95\% tile }\end{array}$ & 1995 Chang \\
\hline Angle of Trunk Joint & $-8^{\circ} \sim 15.6^{\circ}$ & $-5^{\circ} \sim 15^{\circ *}$ \\
Angle of Shoulder Joint & $41.2^{\circ} \sim 75^{\circ}$ & $35^{\circ} \sim 90^{\circ}$ \\
Angle of Elbow Joint & $136.3^{\circ} \sim 175.1^{\circ}$ & $95^{\circ} \sim 180^{\circ}$ \\
Angle of Hip Joint & $32.6^{\circ} \sim 104^{\circ} *$ & $30^{\circ} \sim 80^{\circ}$ \\
Angle of Knee Joint & $54.9^{\circ} \sim 153.3^{\circ}$ & $60^{\circ} \sim 130^{\circ}$ \\
Angle of Ankle Joint & & $78^{\circ} \sim 102^{\circ}$ \\
\hline
\end{tabular}

Remark: ${ }^{*}$ means after adjustment that these two comparable bases can be unified.

riding joints belong to two groups of movable joints, namely 1) functional exercise and 2) dissected exercise. Only functional exercise is discussed in this study, since this is the state of normal joints in exercise. Most articles on human factors typically discuss the movable joints. With regard to comfort, few articles can be found, and they are typically very subjective. Table 3 is a comparison of body joint angles with regard to comfort between Juang ${ }^{12)}$ and Chang ${ }^{2}$. This demonstrates the limitation for the angles of the joints. We will analyze the relation between joint angles and subjective perceptions of comfort to obtain a more objective range for the comfort of joints in the future.

\section{3-2. Subjective Sensation}

One-way analysis of variance (ANOVA) was used to test whether significant changes of any response were dependent on seat height and handlebar height. Significance was accepted at the level of $p<0.05$. In this study, the statistical analysis was run by Win-
Tab. 4 Relationships between body and subjective sensation.

\begin{tabular}{ccc}
\hline & F value & P value \\
\hline hands and arms & $5.093^{* *}$ & 0.003 \\
feet and legs & $6.983^{* * *}$ & 0.000 \\
hip & $26.746^{* * *}$ & 0.000 \\
trunk & $3.231^{*}$ & 0.029 \\
\hline Remark : $p<0.05^{*}$ & $p<0.01^{* *} \quad p<0.001^{* * *}$ \\
& & \\
& & \\
\hline
\end{tabular}

Fig. 1 Total subjective sensations with different seat heights (S 1 S 2 S 3 S 4) and handlebar heights (H 1 H 2).

dows SPSS statistics program.

When riding a bicycle, seat height had a significant effect on the subjective sensations of the hands and arms $(p<0.01)$, feet and legs $(p<0.001)$, hips $(p<$ $0.001)$, and trunk $(p<0.05)$ (Table 4$)$. The subjects' hands and arms felt more uncomfortable at seat height S 4 than at seat height S 2 and S 1. The subjective sensations of the feet and legs showed higher values (more uncomfortable) at seat height S 4 than at other seat height conditions. The values of subjective sensations from the trunk showed higher at seat height S 4 than at seat height S 1. The subject felt more uncomfortable in the hips at seat height S 4 and S 3 than at seat height S 2 and S 1.

When riding a bicycle, the total subjective sensation significantly depends on seat height $(p<0.001)$ and handlebar height $(p<0.05)$. In particular, subjects were more uncomfortable at higher seat heights and at higher handlebar positions (Figure 1).

Psychological responses while riding were affected by seat height. The preferable sensation range could be calculated by the regression equation of the psychological responses. The regression equation is

$$
Y=459.464-1.4531 X+0.0012 X^{2}
$$

and the regression curve is show in Figure 2. The value $Y$ is preferable subjective sensation, and the value $X$ is seat height in the regression equation. 


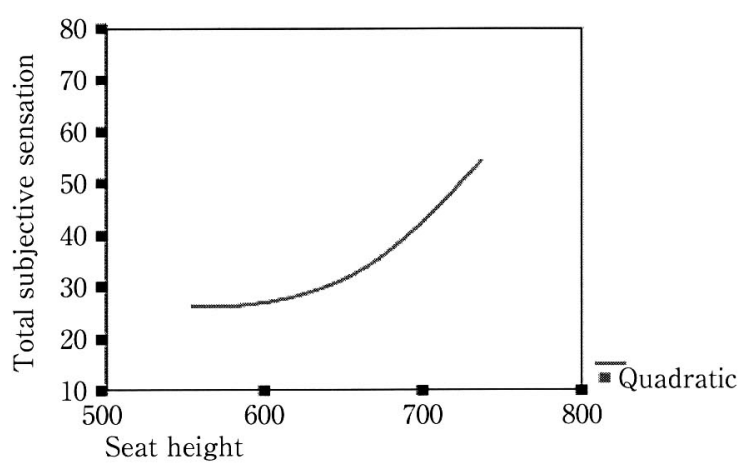

Fig. 2 Regression curve of total subjective sensation.

Using this regression equation, the preferable seat height can be evaluated for comfortable riding posture. The data of this study can also be used to set up the riding simulation model on a computer to help designers improve the ergonomics of a ideally comfortable bicycle.

\section{Discussion}

The psychological responses were affected by seat height and handlebar height during riding bicycle. The subjective sensation during riding bicycle could be estimated by the regression equation of the psychological responses. Using the parameters of body joint angles and regression equation, preferable seat height can be evaluated for comfortable riding posture. Consequently, the studied result can also be implemented in computer simulation model for consultation during selecting and designing of bicycle. Although the standard size of human and bicycle were implemented in computer simulation model, the actual parameter can be modified by individual. The model may be largely attributed to improve the development of ergonomics on bicycle and help design comfortable riding bicycle.

The riding model with computer simulation is based on the anthropometry of bicycle riding and the data bank of riders' psychological responses. The main task of this computer simulation model is to determine the preferable seat heights. We use Auto CAD as the visual screen, Auto LISP language for programming and construction of the parameter design system, and a DCL language program as an interacting speech icon. The computer simulation program fol-

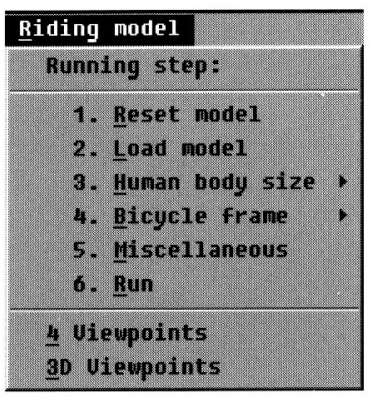

Fig. 3 Items of riding model menu.

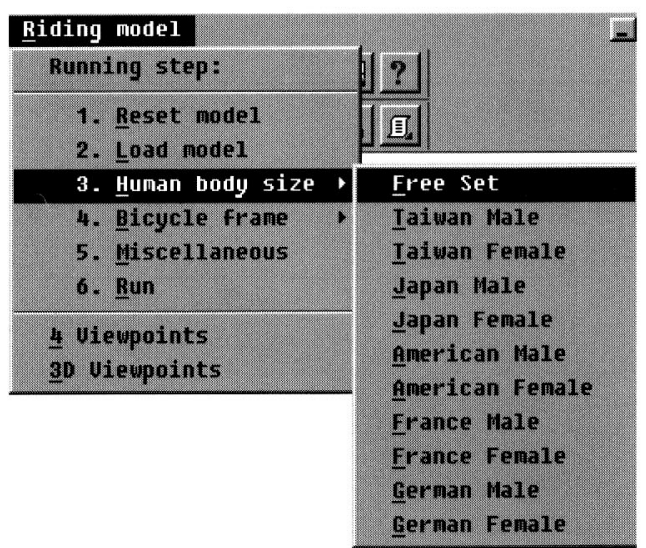

Fig. 4 Choose "Human body size" item on the menu.

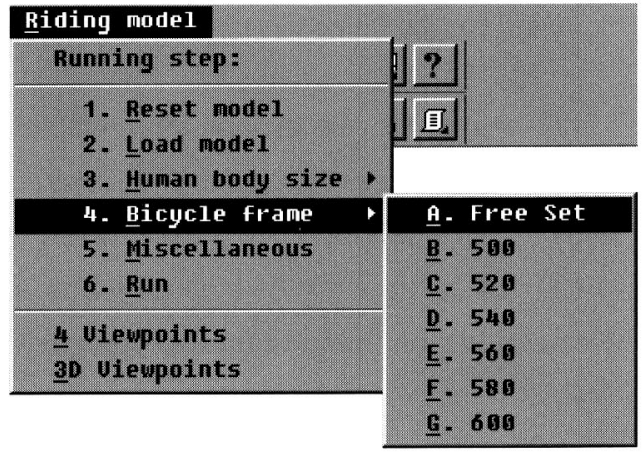

Fig. 5 Choose "Bicycle frame" item on the menu.

lows these steps:

(1) Choose "Load model" in the riding model menu. The computer will transfer the image directly into the AutoCAD system (Figure 3).

(2) Choose "Human body size." There are ten set types of body sizes and one adjustable body size setting (Figure 4).

(3) Choose "Bicycle frame." There are 5 established types of bicycle frames and one adjustable frame (Figure 5).

(4) The "Miscellaneous" menu choice is used to change the display. You may change the procedure 
and image to six different settings.

(5) Choose "Run" to start calculating the best values for seat height and handlebar height.

When we input data of human body size and bicycle frame, the riding posture has been decided. Then, the computer simulation program will create two four bar linkage mechanisms. One is the dynamic motion of the lower body, including thigh, leg, seat tube, and crank to make up a linkage mechanism of lower body, the other is the static motion of upper body which composed of (1) the center of the handlebar, (2) elbow joint, (3) shoulder joint, and (4) the point where the hip is seated. In addition, the angles of knee, hip, trunk, elbow and shoulder with different seat height and handlebar height can be calculated from the assumed linkage mechanisms. Moreover, the calculated results will be checked with objective range for the comfort of joints, like the range of each item in Table 3 , and

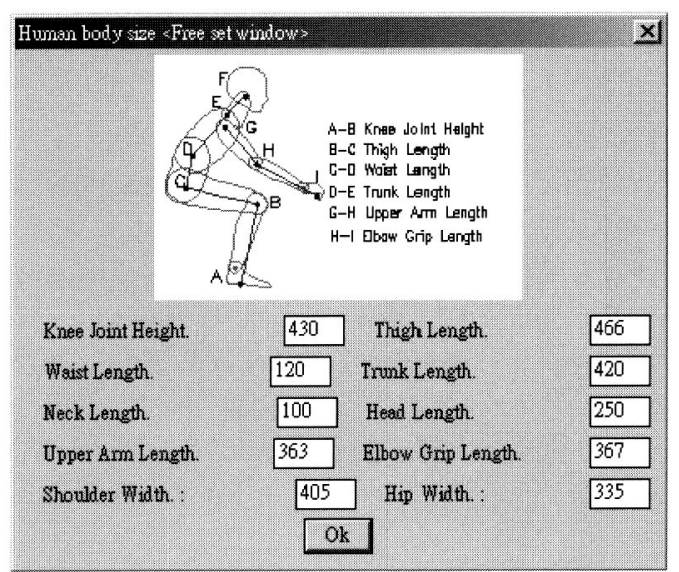

Fig. 6 Values of standard body size.

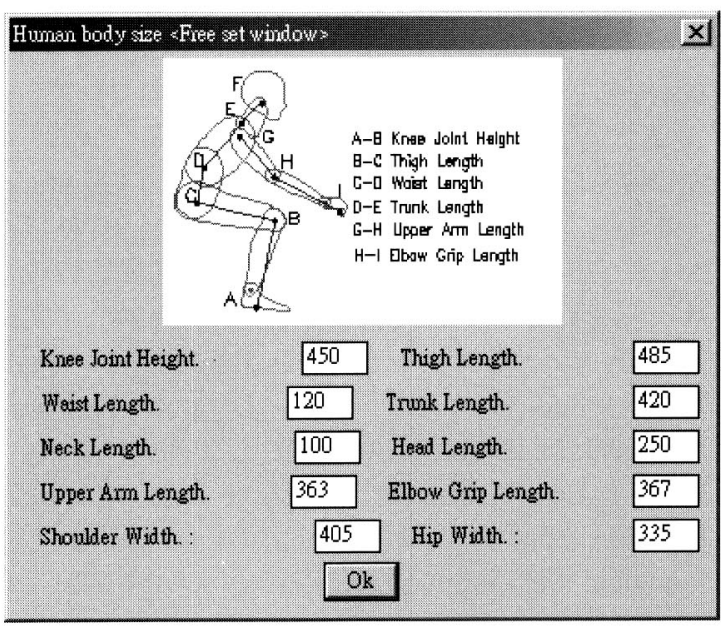

Fig. 7 Values of already changed body size. will decide preferable riding range of seat height and handlebar height. The Borg's values of total subjective sensation can be obtained by inserting the range of seat height to regression equation.

For example, we ran the program with two different body sizes and bicycle sizes. The first time we used a standard body size based on a height of $170 \mathrm{~cm}$ (like Figure 6) and a 500-style bicycle frame. The second time we used an already changed body size (like Figure 7) and a 600-style bicycle frame. Program execution results (Figures 8 and 9) show on the screen display. We will see (1) a man figure riding a bicycle ; (2) the range of preferable seat heights; and (3) the values of subjective sensations. The preferable range of seat heights for the standard body size and 500style frame is 569 to $689 \mathrm{~mm}$. Sometimes the Borg's scale value of subjective sensations are lower than 30 . The Borg's scale value 30 is "uncomfortable." Thus we know the preferable range of seat heights at for this body size and frame type is 569 to $689 \mathrm{~mm}$. For the changed body size and 600-style frame, the prefer-

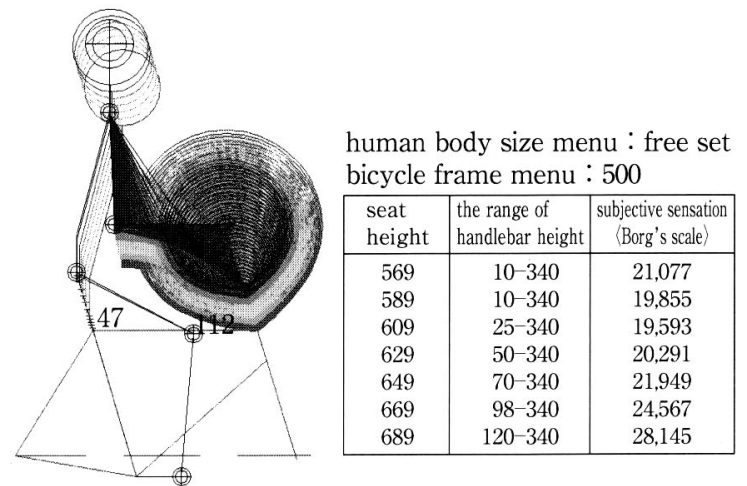

Fig. 8 Output of running results with standard body size and 500-style bicycle frame.

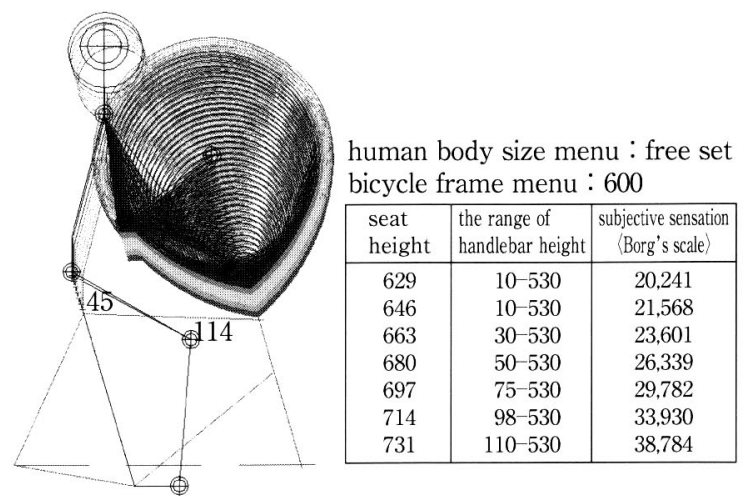

Fig. 9 Output of running results with already changed body size and 600-style bicycle frame. 
able range of seat heights is 629 to $731 \mathrm{~mm}$. Since the Borg's scale value of subjective sensations for this body size and frame is over 30 at seat heights $714 \mathrm{~mm}$ and $731 \mathrm{~mm}$, we can judge the preferable range of seat heights to be 629 to $697 \mathrm{~mm}$.

\section{Conclusion}

In conclusion in this study, we found: (1) The higher seat height, the more uncomfortable and the larger the Borg's values of total subjective sensation at the same time. (2) The Borg's values of total subjective sensation tended to increase with handlebar height. (3) By using the bicycle riding model with computer simulation, we can determine the preferable seat heights, handlebar heights and the Borg's values of total subjective sensation. This will benefit bicycle design. The computer simulation model established by this system will improve the development of ergonomic analysis of bicycles and help design more comfortable bicycles for riding.

The point of our research has been to create a computer simulation model of bicycle-riding to discover the optimal positions for the seat and handlebar for different riders and bicycle frames. We believe that our system is convenient and enjoyable to use. Our system has the following features:

1) When the program is running, it will always display feedback to users to help complete the analysis quickly.

2) It has a highly friendly user interface. The display of icon images conveys information in a fun and accessible way.

3) It has an easy-to-operate parameter design: Interactive windows let users input information fast.

4) The program can investigate the requested calculations under direction from the users based on the accumulation of human body and bicycle frame data and its exercise of information control.

Our system can help to create a specialized bicycle ergonomics system. The direction of follow-up research will continue to explore the relationship between human size and the evaluation of tiredness and subjective sensations to help designers adjust the program parameters and operating interface. Finally, the successes of this computer-simulated bicycle model will be expanded into other models to improve bicycle design, to the benefit of both domestic and foreign markets.

\section{Acknowledgement}

The authors would like to thank the NSC of the Republic of China for financially supporting this research. (Project Nos. NSC-91-2213-E-324-028 and NSC-92-2213-E-324-012)

\section{References}

1) D. Y. Chen, The Subjective Comfort and Physiological Response on Bicycle Riding in Gradient Vibration Environments, Master Paper of NCKU, Tainan : 1999.

2) H.C. Chang, The Computer simulation System of Person-Machine Relationships on Bicycle Riding, Master Paper of NCKU, Tainan : 1995.

3) M. Juan, A Study of Man-Machine Relationships on Bicycle Riding, Master Paper of NCKU, Tainan: 1998.

4) K. Mestdagh, Personal perspective: in search of an optimum cycling posture, Applied Ergonomics, 29(5), 325 334, 1998.

5) H. C. M. Christiaans Henri \& A. Bremne, Comfort on bicycles and the validity of a commercial bicycle fitting system, Applied Ergonomics, 29 (3), 201 211, 1998.

6) A. V. Gunnar, Psychophysical bases of perceived exertion, Medicine and Science in Sports and Exercise, 377 381, 1982.

7) R. A Likert, A technique for the development of attitude scales, Educational and Psychological Measurement, 12, 313 315, 1952.

8) Institute of Occupational Safety \& Health, The Establishment of Static and Dynamic Anthropometry Database of Worker Population (II), IOSH, Taipei : 1997.

9) S. Pheasant, Body space anthropometry ergonomics and design, Taylor \& Francis Inc., London, 1992.

10) A. R. Tilley, The Measure of Man and Woman, Whitney Library of Design, New York, 1993.

11) Research Institute of Human Engineering for Quality Life, Human Body Dimensions Data for Ergonomic Design, Japan Publish Service Co., Tokyo, 1996.

12) M. C. Juang, A study of important anthropometry of bicycle riding, The 8th National Conference on Technological and Occupational Education, 412 421, 1993. 